This item is the archived peer-reviewed author-version of:

MRI following primary repair of the anterior cruciate ligament

\title{
Reference:
}

De Smet Eine, Heusdens C.H.W., Parizel Paul M., Van Dyck Pieter.- MRI follow ing primary repair of the anterior cruciate ligament Clinical radiology / Royal College of Radiologists [London] - ISSN 0009-9260 - London, W b saunders co Itd, 74:8(2019)10 p.

Full text (Publisher's DOI): https://doi.org/10.1016/J.CRAD.2019.03.014

To cite this reference: https://hdl.handle.net/10067/1596400151162165141 


\section{MRI Following Primary Repair of the Anterior Cruciate Ligament}

Eline De Smet ${ }^{1}$, Christiaan H.W. Heusdens ${ }^{2}$, Paul M. Parizel ${ }^{1}$, Pieter Van Dyck ${ }^{1}$

1. Department of Radiology, Antwerp University Hospital and University of Antwerp, Wilrijkstraat 10, 2650 Edegem, Belgium (Tel +32 382135 32; E-mail: elinedesmet@hotmail.com; paul.parizel@uza.be; pieter.vandyck@,uza.be)

2. Department of Orthopedics, Antwerp University Hospital and University of Antwerp, Wilrijkstraat 10, 2650 Edegem, Belgium (Tel +32 382154 83; E-mail: krik.heusdens@uza.be)

Corresponding Author:

Eline De Smet, MD

Department of Radiology

Antwerp University Hospital and University of Antwerp

Wilrijkstraat 10

B-2650 Edegem

Belgium

Tel +3232757043

Fax +32 38252026

E-mail: elinedesmet@hotmail.com 
Funding: This work was supported by the research foundation FWOVlaanderen, Belgium [grant numbers T001017N and $1831217 \mathrm{~N}$ to C.H.W.H. and P.V.D, respectively]. The funders had no role in the design, implementation, or analysis of this research.

Acknowledgement: The authors thank AKIRA Translations (Brussels, Belgium) for language help and translation. 


\section{ABSTRACT}

Primary repair of the anterior cruciate ligament (ACL) is being performed increasingly in the treatment of acute proximal ACL ruptures. Advantages of ACL repair over surgical reconstruction with a tendon graft include preservation of the anatomy and proprioceptive function of the native ACL, and therefore faster rehabilitation. The addition of an internal brace protects the repair during ACL healing and can increase the success rate of the procedure. Given this evolution of ACL surgical treatment, radiologists should be familiar with the new repair techniques and their appearances on postoperative imaging. In this article, we describe two different surgical techniques for primary ACL repair, Dynamic Intraligamentary Stabilization and Internal Brace Ligament Augmentation, and provide an overview of the normal and abnormal appearances after this type of repair at magnetic resonance imaging (MRI) follow-up.

KEY WORDS: Anterior cruciate ligament - ACL repair - ACL healing Dynamic Intraligamentary Stabilization - Internal Brace Ligament Augmentation - Magnetic resonance imaging

1 guarantor of integrity of the entire study: PVD

2 study concepts and design: EDS/CHWH/PVD

3 literature research: EDS/PVD

4 clinical studies: EDS/CHWH/PVD

5 experimental studies/data analysis; N/A 
6 statistical analysis: N/A

7 manuscript preparation: All authors

8 manuscript editing: All authors

\section{HIGHLIGHTS}

(1) There is renewed interest in primary repair of the ACL

(2) Radiologists should be familiar with the normal MRI appearance of ACL healing after repair

(3) Absence of healing on MRI should be interpreted with caution, and clinical correlation is necessary

\section{INTRODUCTION}

Although it was abandoned in the past after arthroscopic ACL reconstruction became popular in the early 1990s, there has been renewed interest in primary ACL repair over the past decade. ${ }^{1}$ This is due to a combination of improved knowledge of the basic biology of human ACL healing 2,3 and the recent availability of new ACL repair techniques. ${ }^{4-6}$ Although the intraarticular ACL fails to heal after complete rupture due to the lack of stable blood clot formation in the gap between the ruptured ends of the ligament, recent studies have shown that the proximal ACL has a surprisingly productive response to injury. ${ }^{7,8}$ Murray et al. reported histologically different zones in the human ACL, with a higher cellular and vascular density in the proximal part of the ligament compared to the distal part. ${ }^{7}$ Nguyen et al. studied human biopsy samples from ACLs that were scarred to 
the posterior cruciate ligament at the time of reconstruction. ${ }^{8}$ These authors showed that the proximal ACL has an intrinsic healing response with typical characteristics similar to the medial collateral ligament that can heal spontaneously. ${ }^{8}$ Consequently, new ACL repair techniques are being developed with focus on the repair of proximal ACL ruptures. $4,6,9,10$ Primary ACL repair has several advantages over reconstruction, including preservation of the native ACL and its proprioceptive function, avoidance of morbidity associated with graft harvesting and incorporation-related issues, and faster recovery. ${ }^{2,4}$ The first clinical results of primary ACL repair are promising. ${ }^{4,6,9,10}$ Published studies have confirmed a definite trend towards improved outcomes in patients with acute proximal ACL rupture because this group tends to have better tissue quality. ${ }^{2,6,9}$ In contrast, poor tissue quality is typical for midsubstance and chronic ACL ruptures, and a repair of these injuries will predictably fail. ${ }^{11,12}$

Few studies published in the orthopedic literature have included MRI in the follow-up of patients after primary ACL repair. ${ }^{13,14}$ However, these studies were primarily concerned with the functional outcomes of the ACL repair, and the MRI findings were not discussed in detail. In particular, longitudinal assessment with MRI of ACL healing following repair with an internal brace has not been well documented. In this article, we describe the surgical techniques for primary ACL repair with Dynamic Intraligamentary Stabilization and Internal Brace Ligament Augmentation, and provide an overview of the normal and abnormal findings after such repair at MRI follow-up. MRI data were collected as part of a pilot study performed prior to 
an ongoing multicenter, randomized controlled trial in our institution comparing ACL repair and conventional ACL reconstruction for relative clinical efficacy and economic benefit. Our Institutional Review Board approved this study and written informed consent was obtained from all participants.

\section{SURGICAL TECHNIQUES}

The goal of primary ACL repair is to reapproximate the proximally ruptured ACL to the lateral femoral condyle and use the proximal part of the ligament's healing capacity. ${ }^{4-6}$ With the emergence of modern arthroscopic surgical instrumentation and implants, several techniques are available for primary ACL repair. Some authors have reported arthroscopic primary ACL repair using suture anchor technology.4,9 Others have augmented the repair with an internal brace to reinforce and protect the repaired ACL during biological healing. If the forces on the healing ACL are too high and could lead to a re-rupture, the augmentation takes over the force. 5,6 Although there is no consensus regarding the single best technique for primary ACL repair, there are indications that some form of internal bracing, with either nonabsorbable suture, scaffolds or a graft, can increase the success rate of the repair procedure. ${ }^{6}$ Two ACL repair techniques facilitating ACL healing, both with a different form of an internal brace, have recently become available for clinical use, namely, InternalBrace ${ }^{\mathrm{TM}}$ Ligament Augmentation (IBLA, Arthrex, Naples, Florida, USA) ${ }^{15,16}$ and Dynamic Intraligamentary Stabilization (DIS or Ligamys $^{\mathrm{TM}}$, Mathys Ltd Bettlach, Switzerland). ${ }^{13}$ The first clinical results obtained with these techniques are promising. ${ }^{17-20}$ 


\section{Internal Brace Ligament Augmentation}

The IBLA procedure (Fig 1) is a static independent suture tape reinforcement technique combining ACL suture repair with ultra-high-strength suture tape (FiberTape) to protect the repair. ${ }^{15,16}$ First, a $3.5 \mathrm{~mm}$ tibial tunnel is drilled, ending at the center of the ACL footprint. The distal part of the ruptured ACL is reapproximated against the lateral femoral condyle or proximal ACL remnant with a lasso suture- and microfracturing of the notch is performed to promote ACL healing. The femoral tunnel is drilled from the center of the femoral footprint inside out. Then, the FiberTape is advanced in the bone tunnels together with the lasso suture and anchored to the bone much like a standard ACL graft (Fig 2). This tape provides further support for the repair during the early healing phase. The IBLA procedure can be carried out for up to 12 weeks following rupture, depending on the ACL remnant length and tissue quality.

\section{Dynamic Intraligamentary Stabilization}

The DIS procedure (Fig 3) provides dynamic posterior tibial translation to protect the repaired ACL. ${ }^{13}$ After primary ACL repair with transosseous absorbable sutures and microfracturing of the notch, the knee is stabilized with a thick polyethylene wire that is passed through the femoral footprint and behind the tibial footprint in order to prevent damaging the ACL's blood and nerve supply. The wire is tensioned by means of a dynamic spring component fixed on the anteromedial aspect of the tibia (Fig 4). The spring, covered with a screw, holds the knee in a constant posterior drawer position at all degrees of flexion, ensuring that the ligament stumps are kept as close 
to each other as possible at all times to facilitate ACL healing. Ideally, the procedure should be performed within 3 weeks after the rupture. This time frame was chosen by the developers of the implant to increase the chance of success of the repair, since after more than 4 weeks the ACL stumps tend to get rounded and at least partially resorbed. Also, the healing potential of a ruptured ACL is expected to reduce over time. ${ }^{3,13}$

\section{MRI OF ACL HEALING AFTER REPAIR WITH IBLA AND DIS}

The MRI appearance of the healing ACL after repair with an internal brace is variable and changes with time. In the first 3 months postoperatively, the ligament has an edematous aspect due to the previous trauma as well as the surgical intervention (Figs. 5 and 6). Between 3 and 6 months after surgery, a gradual decrease of the signal intensity and swelling of the ACL over time occurs, resulting in a normal-sized ACL having low signal intensity within one year after surgery. This time course of MRI changes of the ACL following primary repair with an internal brace can most commonly be observed in patients with a clinically stable knee. This suggests that stability of the knee at this early stage is being maintained not only by the internal brace, but also, at least in part, by the healing of the repaired tissue. ${ }^{14,21}$ Moreover, any case of non-healing would gradually become clinically apparent within the first postoperative year, as there is a gradual loss of the device tension within the first 6 postoperative months. ${ }^{21}$ Therefore, internal bracing techniques rely on healing of the ACL to be effective. Typically, the healing status on MRI remains unchanged between 12 and 24 months postoperatively. 
The time course of changes of the ACL repair contrasts with that commonly observed after standard ACL reconstructions, in which the signal intensity of the ACL graft gradually increases in the early postoperative period, peaks at 4 to 8 months after surgery, and then decreases with further graft maturation (a process referred to as 'ligamentization'). ${ }^{22}$ MRI findings indicate that the healing process of the repaired ACL is different and does not involve such "ligamentization" phase.

Occasionally, high signal intensity of the ACL repair can be seen persisting for up to 12 months or even longer after surgery (Fig. 7). A possible explanation for this observation is the so-called "windshield-wiper effect". ${ }^{23}$ This phenomenon, described in conventional ACL reconstruction, is caused by (sagittal) graft motion with flexion and extension of the knee, which may lead to bone tunnel widening. Similarly, it might be that the internal brace acts as a healing obstacle, resulting in a longer healing process. ${ }^{14}$ Therefore, we suggest that it is not prudent in the first postoperative year to consider the repair to have failed based on increased signal intensity after this type of repair.

\section{COMPLICATIONS}

The most frequently encountered complications after primary ACL repair with internal bracing include absence of healing, arthrofibrosis and rerupture of the ACL repair.

Over time, the ACL can be partially replaced by irregular strands of fibrous tissue bridging between the femur and tibia with extensive scarring of the 
notch (Fig. 8). Typically, these findings show little evolution over time and may be visible on MRI up to 24 months after surgery. It is important to note that absence of healing on MRI is not always accompanied by instability of the knee. This discrepancy between clinical and imaging findings may be explained by both restoration of the ACL's integrity and scar tissue formation, and clinical recovery of ACL function is resultant to both of them. ${ }^{14}$

Arthrofibrosis is a common complication after ACL repair (Fig. 9). It is defined as the presence of excessive scar tissue in the (knee) joint, leading to symptoms of joint stiffness and a painful restriction of motion. ${ }^{24}$ Although the risk factors remain unclear, the timing of surgery is generally considered an important predictor of arthrofibrosis with an increased incidence if surgery is performed within the first four weeks following trauma. ${ }^{24,25}$ As primary repair is performed in acute settings to prevent ligament retraction, arthrofibrosis can be expected a common finding after ACL repair, especially in the case of DIS repair, which is performed within 3 weeks after injury. In addition, microfracturing at the femoral footprint during the ACL repair may also contribute to (excessive) scar tissue formation. Interestingly, local arthrofibrosis (so called 'cyclops lesion'), most commonly attributed to incorrect positioning of the tibial tunnel in conventional ACL reconstruction, can also be seen after primary (anatomical) repair of the ACL (Fig. 10). In patients with symptomatic limitation in the ROM, early arthroscopic arthrolysis (<1year) is needed to improve outcome. ${ }^{25}$ The use of novel biological/tissue engineering techniques, including growth factors, stem cells 
and bio-scaffolds, has been the focus of current research in ACL healing and repair. ${ }^{3}$ Future studies should assess how to guide the biological healing response optimally, as excessive biological activity may lead to hypertrophic scar tissue formation whereas lack of a biological response leads to failure of the ACL repair procedure..$^{2,3,14}$

Rerupture of the ACL repair typically occurs between 8 and 12 months postoperatively, in patients performing highly competitive sports as well as patients with mid-substance tears. MRI findings of complete rerupture include complete discontinuity of the repair with absence of intact ACL fibers, T2-hyperintense fluid in the gap and horizontal fiber orientation or laxity of the ACL (Fig. 11). Hydrops may be present in case of an acute rupture. Clinically, this is accompanied by signs of instability (anterior drawer, Lachman and pivot shift test). Partial rupture of the ACL repair may be more difficult to diagnose as high signal intensity may be present due to prolonged healing. We suggest that any new high MRI signal within the repair should be carefully correlated with the clinical findings. In the case of a repair rupture, a standard ACL reconstruction can still be performed as "no bridges are burned" after primary repair. ${ }^{4,12}$

A well-known late complication of standard ACL reconstruction is the formation of ganglion cysts within the bone tunnels due to degeneration of the tendon graft. ${ }^{26}$ Cystic fluid collections can extend proximally through the tibial tunnel into the joint space or distally into the soft tissues anterior to the tibial tubercle. Although cyst formation in the graft is generally not associated with graft failure, it can cause pain and limitation of motion. In 
our experience, ganglion cysts are not encountered after primary ACL repair as only a $2-\mathrm{mm}$ internal brace is passed through small $(3.5-\mathrm{mm})$ bone tunnels. Similarly, complications associated with graft harvesting can be avoided with the new ACL repair procedures. ${ }^{2,4}$

\section{CONCLUSIONS}

Primary repair of the ACL with internal bracing is being performed increasingly for the treatment of acute proximal ACL ruptures. In this article, we have reviewed the new Dynamic Intraligamentary Stabilization and Internal Brace Ligament Augmentation ACL repair techniques, the normal appearance of the repaired ACL on MRI as well as the possible complications of these techniques. The healing process of the repaired ACL is different compared to ACL reconstruction, without "ligamentization" phase. Although patients may demonstrate signs of ACL healing on MRI following repair, persistent high signal intensity within the repair can be seen for more than 12 months postoperatively in clinically stable knees. Absence of healing on MRI should be interpreted with caution, and correlation with clinical findings is necessary. It is important for the radiologist to be familiar with the normal MRI appearance of ACL healing after repair, and its potential complications in order to avoid misinterpretation and subsequent unnecessary or delayed surgical intervention.

\section{REFERENCES}


1. van der List JP, DiFelice GS. Role of tear location on outcomes of open primary repair of the anterior cruciate ligament: A systematic review of historical studies. Knee 2017;24:898-908. doi: 10.1016/j.knee.2017.05.009.

2. Murray MM, Fleming BC. Biology of anterior cruciate ligament injury and repair: Kappa Delta Ann Doner Vaughn award paper 2013. J Orthop Res 2013;31:1501-1506. doi: 10.1002/jor.22420.

3. Kiapour AM, Murray MM. Basic science of anterior cruciate ligament injury and repair. Bone Joint Res 2014;3:20-31. doi: 10.1302/20463758.32.2000241.

4. Taylor SA, Khair MM, Roberts TR, DiFelice GS. Primary repair of the anterior cruciate ligament: a systematic review. Arthroscopy 2015;31:22332247. doi: 10.1016/j.arthro.2015.05.007.

5. Tapasvi SR, Shekhar A, Patil SS. Primary anterior cruciate ligament repair with augmentation. Arthrosc Tech 2018;7:e139-e145. doi: 10.1016/j.eats.2017.08.063.

6. van Eck CF, Limpisvasti O, ElAttrache NS. Is there a role for internal bracing and repair of the anterior cruciate ligament? A systematic literature review. Am J Sports Med 2017:363546517717956. doi:

$10.1177 / 0363546517717956$.

7. Murray MM, Spector M. Fibroblast distribution in the anteromedial bundle of the human anterior cruciate ligament: the presence of alphasmooth muscle actin-positive cells. J Orthop Res 1999;17:18-27. 
8. Nguyen DT, Ramwadhdoebe TH, van der Hart CP, Blankevoort L, Tak PP, van Dijk CN. Intrinsic healing response of the human anterior cruciate ligament: an histological study of reattached ACL remnants. J Orthop Res 2014;32:296-301.

9. Achtnich A, Herbst E, Forkel P, Metzlaff S, Sprenker F, Imhoff AB, Petersen W. Acute proximal anterior cruciate ligament tears: outcomes after arthroscopic suture anchor repair versus anatomic single-bundle reconstruction. Arthroscopy 2016;32:2562-2569. doi: 10.1016/j.arthro.2016.04.031.

10. DiFelice GS, van der List JP. Clinical outcomes of arthroscopic primary repair of proximal anterior cruciate ligament tears are maintained at midterm follow-up. Arthroscopy 2018;34:1085-1093. doi:

10.1016/j.arthro.2017.10.028.

11. Krismer AM, Gousopoulos L, Kohl S, Ateschrang A, Kohlhof H, Ahmad SS. Factors influencing the success of anterior cruciate ligament repair with dynamic intraligamentary stabilisation. Knee Surg Sports Traumatol Arthrosc 2017;25:3923-3928. doi: 10.1007/s00167-017-4445-6.

12. van der List JP, DiFelice GS. Preservation of the anterior cruciate ligament: a treatment algorithm based on tear location and tissue quality. Am J Orthop (Belle Mead NJ) 2016;45:E393-E405.

13. Eggli S, Kohlhof H, Zumstein M, Henle P, Hartel M, Evangelopoulos DS, Bonel H, Kohl S. Dynamic intraligamentary stabilization: novel technique for 
preserving the ruptured ACL. Knee Surg Sports Traumatol Arthrosc 2015;23:1215-1221. doi: 10.1007/s00167-014-2949-x.

14. Ateschrang A, Ahmad SS, Stöckle U, Schroeter S, Schenk W, Ahrend MD. Recovery of ACL function after dynamic intraligamentary stabilization is resultant to restoration of ACL integrity and scar tissue formation. Knee Surg Sports Traumatol Arthrosc 2018;26:589-595. doi: 10.1007/s00167017-4656-x.

15. Heusdens CHW, Hopper GP, Dossche L, Mackay GM. Anterior Cruciate Ligament Repair Using Independent Suture Tape Reinforcement. Arthrosc Tech. 2018 Jun 18;7(7):e747-e753. doi: 10.1016/j.eats.2018.03.007. eCollection 2018 Jul.

16. Mackay GM, Blyth MJ, Anthony I, Hopper GP, Ribbans WJ. A review of ligament augmentation with the InternalBrace ${ }^{\mathrm{TM}}$ : the surgical principle is described for the lateral ankle ligament and ACL repair in particular, and a comprehensive review of other surgical applications and techniques is presented. Surg Technol Int 2015;26:239-255.

17. MacKay G, Anthony IC, Jenkins PJ, Blyth M. Anterior cruciate ligament repair revisited. Preliminary results of primary repair with internal brace ligament augmentation: a case series. Orthop Muscul Syst 2015;4:188. doi:10.4172/2161-0533.1000188.

18. Heusdens CHW, Hopper GP, Dossche L, Roelant E, Mackay GM. Anterior cruciate ligament repair with Independent Suture Tape Reinforcement: a 
case series with 2-year follow-up. Knee Surg Sports Traumatol Arthrosc 2018 Oct 31. doi: 10.1007/s00167-018-5239-1.

19. Henle P, Röder C, Perler G, Heitkemper S, Eggli S. Dynamic Intraligamentary Stabilization (DIS) for treatment of acute anterior cruciate ligament ruptures: case series experience of the first three years. BMC Musculoskelet Disord 2015;16:27. doi:10.1186/s12891-015-0484-7.

20. Eggli S, Röder C, Perler G, Henle P. Five year results of the first ten ACL patients treated with dynamic intraligamentary stabilisation. BMC Musculoskelet Disord 2016;17:105. doi: 10.1186/s12891-016-0961-7.

21. Kohl S, Evangelopoulos DS, Schär MO, Bieri K, Müller T, Ahmad SS. Dynamic intraligamentary stabilisation: initial experience with treatment of acute ACL ruptures. Bone Joint J 2016;98-B(6):793-798. doi: 10.1302/0301-620X.98B6.35040.

22. Claes S, Verdonk P, Forsyth R, Bellemans J. The "ligamentization" process in anterior cruciate ligament reconstruction: what happens to the human graft? A systematic review of the literature. Am J Sports Med 2011;39:2476-2483. doi: 10.1177/0363546511402662.

23. Clatworthy MG, Annear P, Bulow JU, Bartlett RJ. Tunnel widening in anterior cruciate ligament reconstruction: a prospective evaluation of hamstring and patella tendon grafts. Knee Surg Sports Traumatol Arthrosc. $1999 ; 7(3): 138-145$. 
24. Mayr HO, Weig TG, Plitz W. Arthrofibrosis following ACL reconstructionreasons and outcome. Arch Orthop Trauma Surg 2004;124:518-522. doi:10.1007/s00402-004-0718-x.

25. Ekhtiari S, Horner NS, de Sa D, Simunovic N, Hirschmann MT, Ogilvie R, Berardelli RL, Whelan DB, Ayeni OR. Arthrofibrosis after ACL reconstruction is best treated in a step-wise approach with early recognition and intervention: a systematic review. Knee Surg Sports Traumatol Arthrosc 2017;25:3929-3937. doi: 10.1007/s00167-017-4482-1.

26. Meyers AB, Haims AH, Menn K, Moukaddam H. Imaging of anterior cruciate ligament repair and its complications. AJR Am J Roentgenol. 2010 Feb;194(2):476-84. doi: 10.2214/AJR.09.3200.

27. Lu W, Pauly KB, Gold GE, Pauly JM, Hargreaves BA. SEMAC: Slice Encoding for Metal Artifact Correction in MRI. Magn Reson Med 2009;62:6676. Doi: $10.1002 / \mathrm{mrm} .21967$.

\section{LEGEND TO FIGURES}

Fig. 1-InternalBrace ${ }^{\mathrm{TM}}$ technique, right knee, frontal view, $\mathrm{C}$ Arthrex $\mathrm{GmbH}$. Static augmentation of the ruptured ACL. Proximal ACL suture repair augmented with intraligamentary tape. Note cortical interference screw fixation on the tibial side and cortical button fixation on the femoral side. 
Fig. 2-Radiographic findings after IBLA. Note small bone tunnels (arrowheads), which can barely be seen. Femoral button is parallel to the distal femoral cortex (asterisk).

Fig. 3-Ligamys ${ }^{\circledR}$ technique, left knee, frontal view, OMathys Ltd Bettlach. Dynamic augmentation of the ruptured ACL. Proximal ACL suture repair augmented with intraligamentary braid. Note cortical button fixation on the femoral side and a spring-in-screw mechanism on the tibial side.

Fig.4-Radiographic findings after DIS. The tibial implant consists of a spring (thin arrow) and screw (thick arrow)-mechanism. Note small femoral bone tunnel (arrowhead) and proximal fixation with femoral button (asterisk).

Fig. 5-Normal ACL healing after primary repair with IBLA, as seen on sagittal proton density weighted MR images. A, At 3 months (left), ACL repair is slightly thickened and hyperintense and has indistinct margins due to postoperative edema. At 6 months (right), decrease in signal intensity and remodeling are seen. B, Further remodeling takes place between 12 (left) and 24 (right) months postoperatively. At 24 months, a completely healed ACL is seen. Note FiberTape running through the center of the femoral footprint (arrow). Patient had a clinically stable ACL at all time points.

Fig. 6-Clinically stable ACL healing after primary repair with DIS, as seen on sagittal proton density weighted MR images with use of slice encoding for metal artifact reduction (SEMAC). ${ }^{27} \mathbf{A}$, At 3 months (left), ACL repair is hyperintense due to postoperative edema. Note large joint effusion. MR at 6 
months postoperatively (right) shows normal ACL healing exhibiting gradual decrease in the repair's signal intensity. B, Follow-up MR images at 12 (left) and 24 (right) months postoperatively show further gradual decrease in signal intensity with normal continuity of ACL fibers

Fig. 7-Persistent high signal in the ACL after primary repair with IBLA, as seen on sagittal proton density weighted MR images. A, At 6 months, ACL repair is hyperintense due to postoperative edema. High signal intensity of the repair persists at 12 (B) and 24 (C) months postoperatively.

Fig. 8-Clinically stable ACL after primary repair with DIS without signs of healing on MRI. Sagittal proton density weighted MR image at 6 months (A) postoperatively shows irregular appearance of the ACL with abnormal ligament strands and extensive fibrosis at the intercondylar notch. Follow-up MRI assessments at 12 (B) and 24 (C) months postoperatively remain unchanged.

Fig. 9-Decreased ROM after DIS repair due to arthrofibrosis. Sagittal proton density weighted MR images at 12 months postoperatively (left) show extensive fibrosis in Hoffa's fat pad (arrow). Follow-up MRI assessment at 24 months postoperatively (right) shows status after arthroscopic arthrolysis with resection (asterisk) of Hoffa's fat pad. Note normal healing of the ACL repair.

Fig. 10-Cyclops lesion after DIS repair. Axial T2 weighted image with fat suppression (left) and sagittal proton density weighted image (right) reveal a small, oval mass anterior to the ACL repair (arrow) in keeping with localized 
fibrosis/cyclops lesion. Also note the augmentation wire posterior of the tibial ACL footprint (arrowhead).

Fig. 11-Re-rupture of the ACL after repair with IBLA, as seen on sagittal proton density weighted MR images. Follow-up MR images at $3(\mathbf{A}), 6(\mathbf{B})$ and 12 (C) months postoperatively show healing with continuity of the ACL and slightly high signal persisting over time. Two weeks later, patient had a traumatic re-rupture of the ACL repair. New high signal (arrow) is seen in the proximal third of the repaired ligament below the repair construct (D). Also note large joint effusion due to new trauma. 